\title{
A COHOMOLOGICAL CHARACTERIZATION OF FINITE NILPOTENT GROUPS ${ }^{1}$
}

\author{
W. J. WONG
}

In proving the existence of outer automorphisms of finite $p$-groups [2], Gaschütz showed that if $A$ is a finite module with $p$-power order over a finite $p$-group $G$ such that $H^{1}(G, A)=0$, then $H^{n}(H, A)=0$ for all $n \geqq 1$ and all $H \leqq G$. In this note (whose origin lies in some remarks made by Professor Hans Zassenhaus) ${ }^{2}$ we use Gaschütz's method to prove a similar result valid for all finite nilpotent groups and show that this result fails for every finite nonnilpotent group so that we have a characterization of the class of finite nilpotent groups.

We let $H^{n}(G, A)$ denote the $n$th cohomology group of $G$ in $A$ in the sense of Tate (cf. [6]). We recall the method of dimension-shifting. If $A$ is any $G$-module and $Z(G)$ is the group ring of $G$ over the ring of integers, then $Z(G) \otimes A$ is a $G$-module in a natural way, and there exist exact sequences of $G$-modules

$$
\begin{aligned}
& 0 \rightarrow A \rightarrow Z(G) \otimes A \rightarrow B \rightarrow 0, \\
& 0 \rightarrow C \rightarrow Z(G) \otimes A \rightarrow A \rightarrow 0 .
\end{aligned}
$$

Now, $Z(G) \otimes A$ is $G$-regular so that $H^{n}(G, Z(G) \otimes A)=0$ for all $n$, and we have

$$
H^{n}(G, A) \cong H^{n-1}(G, B) \cong H^{n+1}(G, C)
$$

for all $n$. When $A$ is finite then $Z(G) \otimes A$ is finite, so that $B$ and $C$ are also finite.

Theorem 1. Let $G$ be a finite nilpotent group and $A$ a finite G-module such that $H^{m}(G, A)=0$ for some $m$. Then $H^{n}(G, A)=0$ for all $n$.

Proof. If $A_{p}$ is the Sylow $p$-subgroup of $A$, then $H^{n}(G, A)$ $=\sum H^{n}\left(G, A_{p}\right)$, where the direct sum is taken over all primes $p$. It follows that we may assume that $A$ is a $p$-group for some prime $p$. By dimension-shifting, we may assume that $m=1$.

The result is valid if $p$ does not divide $|G|$; in particular, it is valid when $|G|=1$. Thus we use induction on $|G|$ and may assume that $p$ divides $|G|$. Since $G$ is nilpotent, we can find a normal subgroup $H$ of index $p$ in $G$. We have the exact sequence [3, p. 129]:

Received by the editors March 1, 1967.

${ }^{1}$ Research supported by NSF grant GP-3986 at the University of Notre Dame.

${ }^{2}$ Professor Zassenhaus has since informed me that he, K. Hoechsmann and P. Roquette have also obtained the results of this note. 


$$
0 \rightarrow H^{1}\left(G / H, A^{H}\right) \rightarrow H^{1}(G, A) \rightarrow H^{1}(H, A)^{G / H} \rightarrow H^{2}\left(G / H, A^{H}\right) .
$$

Since $H^{1}(G, A)=0$ by hypothesis, we have $H^{1}\left(G / H, A^{H}\right)=0$. Since $G / H$ is cyclic and the Herbrand quotient of the finite module $A^{B}$ is 1 [1, p. 57], we have

$$
H^{n}\left(G / H, A^{H}\right)=0, \text { all } n .
$$

It follows that $H^{1}(H, A)^{G / H}=0$. Since $G / H$ and $H^{1}(H, A)$ are $p$ groups, this implies that $H^{1}(H, A)=0$. By induction hypothesis, we have

$$
H^{n}(H, A)=0, \text { all } n .
$$

Now we have the inflation-restriction and transfer-deflation exact sequences [3, p. 129], [6, p. 321]:

$$
\begin{array}{rlrl}
H^{n}\left(G / H, A^{H}\right) & \rightarrow H^{n}(G, A) \rightarrow H^{n}(H, A), & & n \geqq 1, \\
H^{n}(H, A) & \rightarrow H^{n}(G, A) \rightarrow H^{n}\left(G / H, A^{H}\right), & n \leqq 0 .
\end{array}
$$

From (1) and (2) it now follows that $H^{n}(G, A)=0$, all $n$.

TheOREM 2. Let $G$ be a finite nonnilpotent group, $n$ any integer. Then there exists a finite $G$-module $A$ such that $H^{n}(G, A) \neq 0, H^{n+1}(G, A)=0$.

Proof. By dimension-shifting, we may assume that $n=0$. Let $H$ be a minimal nonnilpotent subgroup of $G$. By a theorem of Schmidt and Golfand [4, p. 304], the commutator subgroup of $H$ is a Sylow $p$-subgroup of $H$ for some prime $p$. Let $B$ be the trivial $H$-module of order $p$. Then,

$$
H^{0}(H, B) \cong B \neq 0, \quad H^{1}(H, B) \cong \operatorname{Hom}(H, B)=0 .
$$

Now let $A$ be the $G$-module induced by $B$ (denoted $M_{G}^{H}(B)$ in $[\mathbf{5}$, p. 1-12]). Then $H^{n}(G, A) \cong H^{n}(H, B)$ for all $n$, so that

$$
H^{0}(G, A) \neq 0, \quad H^{1}(G, A)=0 .
$$

Moreover, $A$ is finite since $B$ is finite.

REMARK. If $G$ is a finite $p$-group and $A$ a finite $G$-module such that $H^{m}(G, A)=0$ for some $m$, then $H^{n}(H, A)=0$ for all $n$ and all subgroups $H$ of $G$, by the proof of Theorem 1, since every proper subgroup of $G$ is contained in one of index p. However, this statement is false whenever $G$ is not a $p$-group. For, suppose that $G$ is a finite nilpotent group whose order is divisible by at least two primes. Let $p$ be one of these prime divisors of $|G|$ and let $H$ be a Sylow $p$-subgroup of $G$. Since $H$ is normal in $G$ and $G / H$ is a nontrivial $p^{\prime}$-group, there exists a nontrivial irreducible $K(G)$-module $A$ on which $H$ acts triv- 
ially, where $K$ is a finite field of characteristic $p$ and $K(G)$ is the group algebra of $G$ over $K$. Then $H^{0}(G, A)=0$ but $H^{0}(H, A) \cong A \neq 0$. Theorem 1 shows that in fact $H^{n}(G, A)=0$ and $H^{n}(H, A) \neq 0$, all $n$.

\section{REFERENCES}

1. C. Chevalley, Class field theory, Nagoya University, Nagoya, 1954.

2. W. Gaschütz, Kohomologische Trivialitäten und äussere Automorphismen von p-Gruppen, Math. Z. 88 (1965), 432-433.

3. G. Hochschild and J.-P. Serre, Cohomology of group extensions, Trans. Amer. Math. Soc. 74 (1953), 110-134.

4. L. Rédei, Die endlichen einstufig nichtnilpotenten Gruppen, Publ. Math. Debrecen 4 (1956), 303-324.

5. J.-P. Serre, Cohomologie galoisienne, Springer, Berlin, 1965.

6. E. Weiss, $A$ deflation map, J. Math. Mech. 8 (1959), 309-329.

UNIVERSITY OF Notre DAME 